\title{
Ziele, Motivationen, Beziehungsorientierung und Fachlichkeit von Nachwuchs- und Spitzenkräften in nachhaltigkeitswissenschaftlich ausgerichteten Arbeitsbereichen. \\ Ergebnisse aus dem Projekt "Nachhaltigkeitskompetenz und Geschlecht" (NaGe) ${ }^{1}$
}

\begin{abstract}
Zusammenfassung
Der Artikel stellt Ergebnisse aus der Analyse von Zielen und Motivationen sowie der Bedeutung sozialer und fachlicher Kompetenzen von Nachwuchs- und Spitzenkräften in wissenschaftlichen, nachhaltigkeitsorientierten Tätigkeitsfeldern vor. Aus Gendersicht wurden Zusammenhänge identifiziert, die auf Menschen und deren Möglichkeiten, an Spitzenpositionen zu gelangen, einwirken. Dabei zeigen sich zwischen Spitzen- und Nachwuchspositionen Verschiebungen in genderkodierten Trennungslinien, die Berufswege beeinflussen können. Diese beziehen sich auf das Verhältnis von Fachlichkeit und Beziehungsorientierung sowie von privat und öffentlich: Während Spitzenkräfte Sozialität instrumentell in den Dienst des Erwerbs von Fachkompetenz stellen, platzieren Nachwuchskräfte das soziale Miteinander im Zentrum und als existenzielles Element ihres Arbeitskontextes. Von den Nachwuchskräften wird weiterhin ein Verhältnis von Erwerbstätigkeit und Privatleben konstruiert, in dem das Private machtvoll ist und aktiv gewählt wird, während Spitzenkräfte im Interview eher die Erwerbsarbeit fokussieren.
\end{abstract}

\section{Schlüsselwörter}

Nachhaltigkeit, Nachhaltigkeitskompetenz, Nachhaltigkeitswissenschaften, geschlechtsspezifische Segregation, Wissenschaft, Spitzenpositionen

\begin{abstract}
Summary
Aims, motivations, relationship-orientation and expertise of junior scientists and professionals in the sustainability sciences. Findings from the project "Nachhaltigkeitskompetenz und Geschlecht" (NaGe)

The article presents results of the analyses of aims, motivations and meanings of social communicative competency and expert knowledge of junior scientists and professionals in scientific occupations for sustainability. The contribution identifies relations and influences which affect people and their possibilities to reach top positions from the perspective of gender. This reveals gender differences between professionals and junior scientists which can affect their further career. It refers to the relationship between expert knowledge and relationship-orientation and between private and public: Professionals instrumentally raise the issue of sociality in an attempt to expert knowledge. Junior scientists transfer relationship-management in the focus of their working contexts. They construct a specific relationship between employment and privacy which is self-selected and powerful in contrast to the professionals who focus on employability.
\end{abstract}

\section{Keywords}

sustainability, sustainability sciences, competencies for sustainability, gender gaps, top positions

1 Das Projekt wird vom Bundesministerium für Bildung und Forschung sowie dem Europäischen Sozialfonds der Europäischen Union unter dem Förderkennzeichen FKZ 01FP1068/69 gefördert und ist am Institut für Nachhaltigkeitssteuerung, Forschungsgebiet Umweltplanung der Leuphana Universität Lüneburg angesiedelt. 


\section{Einleitung}

Auch wenn inzwischen viele ex- und implizite Ausgrenzungs- und Ausschlussmechanismen identifiziert und von ihren Bedingungszusammenhängen her untersucht wurden, ist bis heute nicht umfassend geklärt, woran es liegt, dass für Frauen trotz veränderter individueller, gesellschaftlicher und organisationeller Ausgangssituationen die Aussichten, an Spitzenpositionen zu gelangen, weiterhin schlechter stehen als für Männer (Osterloh/ Littmann-Wernli 2000: 125ff.; Morrison/White/Van Velsor 1987: 8ff.). Die Arbeitswelt ist weiterhin in großen Teilen geschlechterspezifisch segregiert (Müller 2013: 471).

Auch in Institutionen in Deutschland, die (wissenschaftlich) zu Themen einer nachhaltigen Entwicklung arbeiten, findet sich das Phänomen des ,glass ceiling“-Effekts. ${ }^{2}$ So wird das asymmetrische Geschlechterverhältnis in diesem Arbeitsfeld etwa deutlich, wenn beispielsweise Anteile von Männern und Frauen in Spitzen- und Nachwuchspositionen in Projekten betrachtet werden, die im Rahmen von an Nachhaltigkeit orientierten Förderprogrammen des Bundesministeriums für Bildung und Forschung (BMBF) und der Deutschen Forschungsgemeinschaft (DFG) realisiert werden. ${ }^{3}$ Lag hier im Jahr 2011 der Anteil der Nachwuchswissenschaftlerinnen je nach Förderprogramm zwischen 45 und 47 Prozent, betrug der Frauenanteil bei Spitzenpositionen jeweils nur zwischen 11 und 20 Prozent (Katz/Thiem 2011: 135). Ein Mangel an qualifizierten Frauen als Begründung für deren Unterrepräsentanz in Spitzenpositionen kann angesichts des Frauenanteils beim wissenschaftlichen Nachwuchs für diesen Tätigkeitsbereich nicht behauptet werden. Auch ist der Anteil von Frauen in nachhaltigkeitsorientierten Studiengängen hoch (Katz/Thiem 2011: 135ff.). ${ }^{4}$

Diese Befunde sind für nachhaltigkeitsorientierte wissenschaftliche Berufsfelder insofern auffällig, weil entsprechende Organisationen in einem Bereich tätig sind, der sich an einem normativ ausgerichteten Leitbild orientiert, das Geschlechtergerechtigkeit als wichtigen Bestandteil des intragenerativen Gerechtigkeitspostulats impliziert (vgl. Bundesministerium für Umwelt, Naturschutz und Reaktorsicherheit o. J.: Kap. 24). Daher stellt sich die Frage nach konkreten Begründungszusammenhängen für das Fehlen und Vorhandensein von Männern und Frauen in Spitzenpositionen im Kontext wissenschaftlicher Tätigkeiten im Bereich Nachhaltigkeit. ${ }^{5}$

Forschungsstände zu ,Nachhaltigkeit und Geschlecht', ,Kompetenz und Geschlecht‘ sowie ,Organisation und Geschlecht' liefern dazu Hinweise und Anknüpfungspunkte (Katz et al. 2014). Dennoch ist noch weitestgehend offen, ob und inwieweit im Kontext

2 Zum "glass ceiling"-Effekt in der Wissenschaft und an Hochschulen vgl. Kreckel (2005) sowie Blättel-Mink/Briken/Rau (2010).

3 Folgende Forschungsförderprogramme wurden aufgrund ihrer sozial-ökologischen Schwerpunktsetzungen ausgewählt, die damit über eine naturwissenschaftlich-technische Sichtweise auf Nachhaltigkeit hinausgehen: Sozial-ökologische Forschung (BMBF), Nachhaltiges Wirtschaften - Regionale Ansätze (BMBF), Mensch und globale Umweltveränderungen (DFG), Kulturlandschaftsforschungsprogramm des Österreichischen Forschungsministeriums.

4 Im Sommersemester 2010 studierten an der Leuphana Universität Lüneburg beispielsweise 127 Studentinnen (212 Studierende gesamt) den Leuphana-Bachelor mit dem Major Umweltwissenschaften, 23 Studentinnen (43 Studierende gesamt) den Master Sustainability Sciences und 49 Studentinnen (107 Studierende gesamt) Diplom-Umweltwissenschaften.

5 Uta von Winterfeld (2013) stellt vor diesem Hintergrund die Frage nach Veränderungen und Veränderungspotenzialen von Führungsfrauen im Kontext von nachhaltiger Entwicklung. 
wissenschaftlich orientierter Tätigkeiten im Nachhaltigkeitsbereich sich möglicherweise unterscheidende Kompetenzprofile, lebensweltliche und berufsbiografische Kontexte, Wertorientierungen oder unterschiedliche Tätigkeits- und Karrierevorstellungen von Männern und Frauen sowie organisationsstrukturelle und -kulturelle Bedingungen für das Fehlen von Frauen in Spitzenpositionen ausschlaggebend sind (Katz/Thiem 2013). Ziel des Projekts „Nachhaltigkeitskompetenz und Geschlecht“ ist es daher, entsprechende Zusammenhänge zu untersuchen und Faktoren zu identifizieren, die mit der Besetzung von Spitzenpositionen in Beziehung stehen und damit Geschlecht als vertikale Segregation (mit)bedingende Kategorie relevant werden lassen. Das Projekt geht dabei von Geschlecht als sozialer Kategorie aus. Im Mittelpunkt der Betrachtung steht die Wirkung der symbolischen Geschlechterordnung auf das Handeln der Befragten in Interdependenz mit den strukturellen Verhältnissen (Becker-Schmidt 2007 [1993], Hofmeister/Katz/Mölders 2013: 50ff.). Es geht also weniger darum, wissenschaftliche Grundlagen zu schaffen, um Maßnahmen zu entwickeln, die darauf zielen, den Frauenanteil in entsprechenden Positionen zu erhöhen. Vielmehr geht es um die Identifikation und Förderung von Kompetenzen und Strukturen, die es Menschen ermöglichen, in Spitzenpositionen zu gelangen und in diesen nachhaltigkeits- und genderkompetent handeln zu können. ${ }^{6}$ Im Fokus stehen dabei einerseits Fähigkeiten, Ziele und Motivationen und andererseits organisationsstrukturelle und -kulturelle Zusammenhänge, die für nachhaltigkeitsrelevante Tätigkeitsbereiche und den Aufstieg in verantwortungsvolle Positionen bedeutend sind. Im vorliegenden Beitrag geht es darum, aufzuzeigen, wie Arbeitskontexte mit neuen Motiven besetzt werden und dabei Geschlechterverhältnisse und -zuschreibungen re-figuriert und/oder brüchig werden.

\section{Methoden}

Die hier vorgestellten Ergebnisse basieren auf Interviews mit Spitzen- und Nachwuchskräften in wissenschaftlich ausgerichteten Tätigkeitsfeldern im Bereich Nachhaltigkeit. Insgesamt wurden Interviews mit zehn Nachwuchs- (davon sechs Frauen und vier Männer) und fünf Spitzenkräften (davon zwei Männer und drei Frauen) aus drei Organisationen in Deutschland und der Schweiz ausgewertet, mit denen das Forschungsprojekt „Nachhaltigkeitskompetenz und Geschlecht“" kooperiert.

Die kooperierenden Organisationen sind im Bereich nachhaltige Entwicklung angesiedelt, sie decken Tätigkeitsfelder ab, in denen wissenschaftlich zu Themen einer nachhaltigen Entwicklung gearbeitet wird. Ein weiteres Auswahlkriterium war das Selbstverständnis der jeweiligen Organisation, zu einer nachhaltigen Entwicklung beitragen

$6 \quad$ Von Winterfeld (2013) zeigt anhand von Interviews mit Spitzenkräften im Nachhaltigkeitskontext neben Veränderungspotenzialen durch mehr Frauen in Spitzenpositionen auch Sichtweisen auf, wonach ein Mehr an Spitzenfrauen alleine nicht ausreicht, um Nachhaltigkeit zu realisieren. Ferner stellt sie dar, dass Spitzenpositionen Frauen auch verändern können oder nur bestimmte Frauen in diese Positionen gelangen, die der Logik von Spitzenpositionen entsprechen. In diesem Zusammenhang wird auf die Notwendigkeit eines Abbaus von Hierarchien verwiesen, da sich "nachhaltige Ziele mit dem Denken in Spitzen und Hierarchien als kaum vereinbar [erweisen]" (Winterfeld 2013: 196f.). 
zu wollen und die Arbeit daran auszurichten. Die interviewten Spitzenkräfte sind je nach Größe und Struktur der Organisation auf der ersten bis dritten Hierarchieebene angesiedelt, haben Leitungsfunktionen inne und sind für Arbeitsbereiche verantwortlich, in denen u. E. ein gesellschaftliches Handlungsfeld unter der Perspektive des Leitbilds einer nachhaltigen Entwicklung im Fokus steht. ${ }^{7}$ Die Nachhaltigkeitsperspektive meint hier im Kontext des Projekts eine mehr oder weniger stark ausgeprägte Orientierung an den diesem Leitbild zugrunde liegenden Werten (s. o.) und eine integrative Sichtweise auf das jeweilige Thema: Ökonomische, ökologische, soziale (und kulturelle) Aspekte werden integrativ betrachtet, um der Komplexität der unter Nachhaltigkeitsperspektive betrachteten Handlungsfelder und/oder Problemlagen gerecht zu werden (Becker/Jahn 2006). Je nach Organisation umfassen diese Arbeitsbereiche neben einem wissenschaftlichen Fokus auch Tätigkeiten, die stärker auf Realisierungen in der Praxis verweisen.

Die Nachwuchskräfte wurden aus darunter liegenden Hierarchieebenen ausgewählt, in denen eine gezielte Förderung und damit ein Aufstieg und eine weitere Qualifizierung möglich sind. ${ }^{8}$ Ihr Aufgabenspektrum reicht von der alleinigen Verantwortlichkeit für ein Themenfeld, Projekt oder die eigene Dissertation über das Eingebundensein in ein Forschungsteam bis hin zur Leitung kleinerer Arbeitsgruppen und bewegt sich thematisch ebenfalls im Kontext einer nachhaltigen Entwicklung (s. o.).

Im Fokus der 90-minütigen, leitfadengestützten Interviews (Meuser/Nagel 1991, 2004) stehen die zuvor erhobenen Kompetenz- und Werteprofile der entsprechenden Personen. ${ }^{9}$ Die Kompetenzprofile wurden mit dem Kompetenzentwicklungs- und Diagnostiktool KODE ${ }^{\circledR}$ (Heyse/Erpenbeck/Ortmann 2010; auch Erpenbeck 2004; Erpenbeck/ von Rosenstiel 2007 [2003]) ermittelt. ${ }^{10}$

Karl Kreuser (2010) hat in seiner Forschungsarbeit auch den Gender Bias des Verfahrens $\mathrm{KODE}^{\circledR}$ kritisch beleuchtet. Als ein Ergebnis seiner Analyse stellt Kreuser fest, dass das Instrument ,die Frage nach den vorhandenen Ausprägungen der für eine

7 Diese Spitzenkräfte haben bspw. eine wissenschaftliche Projektleitung inne (Verbundprojekt), besetzen eine leitende Stelle im Bereich Wissenschaftsmanagement (Programmgestaltung, Koordination etc.), haben Personalverantwortung oder vertreten eine Position mit repräsentativ ausgerichteten Gestaltungsfunktionen.

8 Zu den Nachwuchskräften gehören Promovierende und Nachwuchswissenschaftler*innen in der Postdoc-Phase (mit Stipendium oder als wissenschaftliche Angestellte) sowie Habilitierende (Stipendium, wissenschaftliche Angestellte, auch als Nachwuchsgruppenleitungen und Juniorprofessor*innen).

9 Das qualitativ erhobene Material wurde in Anlehnung an das Kodierschema von Glaser und Strauss (1998) ausgewertet. Die Auswertung des Datenmaterials fußt auf der Vorgehensweise eines rekonstruktiven texthermeneutischen Verfahrens, einem qualitativen Verfahren der Kodierung und Kategorienbildung qua Kontrastierung von Datenmaterialien.

10 Das mit diesem Verfahren zugrunde gelegte Kompetenzmodell von Heyse und Erpenbeck (2010) basiert auf einem konstruktivistischen Selbstorganisationsmodell, mit dem Kompetenzen als Selbstorganisationsdispositionen verstanden werden, die sich durch die Realisierung in gedanklichem und gegenständlichem Handeln zeigen. Das WERDE ${ }^{-}$-System dient der Ermittlung jener Wertarten, die für Grundkompetenzen bei einer Person handlungsleitend sind. Beide Verfahren beziehen sich auf vier Basiskompetenzbereiche (personale Kompetenzen, aktivitäts- und handlungsbezogene Kompetenzen, fachlich-methodische Kompetenzen sowie soziale Kompetenzen) und insgesamt 16 dazugehörige Teilkompetenzen (Heyse 2010: 81ff.). Die ermittelten Kompetenz- und Werteprofile basieren auf einer Selbstbeurteilung: Mit KODE® wird das Ausprägungsverhältnis der Grundkompetenzen einer Person - sowohl unter normalen, unproblematischen als auch unter besonders fordernden, schwierigen Lebens- und Arbeitsbedingungen - erhoben und abgebildet. 
Aufgabe erforderlichen Kompetenzen ohne Ansehen des Geschlechts beantwortet" (Kreuser 2010: 206f.) und somit für den Einsatz zur Kompetenzerfassung und -entwicklung unter Genderaspekten geeignet ist. ${ }^{11}$

Für den vorliegenden Artikel wurden Aussagen der Spitzen- und Nachwuchskräfte zu Zielen, Motivationen sowie zur Bedeutung und Bewertung von fachlich-methodischen und sozial-kommunikativen Kompetenzen ausgewertet.

\section{Wissenschaftlich ausgerichtete Tätigkeitsfelder im Bereich Nachhaltigkeit: Geschlechterkonnotationen und geschlechterkategoriale Segregation zwischen Kontinuität und Wandel?}

In der Auswertung der qualitativ erhobenen Daten werden Unterschiede zwischen den befragten Gruppen der Spitzen- und Nachwuchskräfte sichtbar, die mit einer Verschiebung genderkodierter Trennungslinien einhergehen. Geschlechterspezifische Segregation befördernde Bedingungs- und Bedeutungszusammenhänge im Kontext wissenschaftlicher Tätigkeitsfelder im Nachhaltigkeitsbereich scheinen hier auf der Ebene geschlechtlich aufgeladener Tätigkeitsbereiche und Positionen zum Ausdruck zu kommen. Auffällig sind vor diesem Hintergrund vor allem zwei Aspekte:

Erstens konstruiert ein Teil der befragten Nachwuchskräfte im Interview ein Spannungsfeld zwischen privaten und beruflichen Zielen, indem sie Prioritäten setzen und Entscheidungen treffen. Private Ziele werden dabei als zentraler Aspekt von Lebensqualität hervorgehoben, eingefordert und entweder den beruflichen Zielen übergeordnet oder als gleichwertig beschrieben. Die Spitzenkräfte gehen demgegenüber in ihren Ausführungen nicht auf private Ziele ein. Bezug genommen wird hier fast ausschließlich auf berufliche Ziele.

Zweitens wird deutlich, dass sowohl Spitzen- als auch Nachwuchskräfte im Kontext ihrer Tätigkeit im Nachhaltigkeitsbereich Fachlichkeit und Beziehungsorientierung jeweils eine hohe Bedeutsamkeit beimessen. Jedoch finden sich hier deutliche Unterschiede: So konstruieren die interviewten Spitzenkräfte Beziehungsorientierung für ihren Arbeitsalltag als zentral, um Fachlichkeit zu generieren. Die Nachwuchskräfte skizzieren im Interview dagegen ein Verständnis von Beziehungsorientierung, das über die Zuordnung zu einer instrumentellen Sichtweise hinausreicht.

In den folgenden Abschnitten sollen diese Zusammenhänge näher beleuchtet und damit verbundene geschlechterspezifische Segregation befördernde Bedingungs- und Bedeutungszusammenhänge, aber auch Kontexte identifiziert werden, in denen sich ein Wandel abzeichnet und Geschlecht als Kategorie an Relevanz verliert.

11 Zur Integration von Genderaspekten wurden im Rahmen des Forschungsprojektes NaGe neben der Erhebung der Ist-Kompetenzprofile, die im Fokus dieses Beitrags stehen, auch genderorientierte Anforderungsprofile für Nachhaltigkeitskompetenzen erarbeitet. Hierfür wurde u. a. ein Anforderungsprofil für Genderkompetenz erstellt und mit den Soll-Profilen für Nachhaltigkeitskompetenzen zusammengeführt. 


\subsection{Zum Verhältnis beruflicher sowie privater Ziele und Motivationen von Spitzen- und Nachwuchskräften: Reproduktion und Auflösung geschlechtsspezifischer Hierarchisierungen}

Inwieweit weisen die in den Interviews konstruierten Verhältnisse zwischen beruflichen und privaten Zielen nun Genderkodierungen auf, die für die Möglichkeiten von Menschen, an Spitzenpositionen zu gelangen, relevant sind? Um ein Analyseraster aufzuspannen, bietet sich hier zunächst ein Rekurs auf die von feministischer Seite kritisierte Trennung zwischen Privatleben und Erwerbstätigkeit an.

Die feministische Forschung hat aufgezeigt, dass und wie Erwerbssphäre und Privatleben bzw. Erwerbsarbeit und Hausarbeit als voneinander abgetrennte Bereiche betrachtet und gelebt werden und inwieweit diese Aufteilung des gesellschaftlichen Lebens mit Geschlechterrollen, Geschlechterverhältnissen und Zuschreibungspraktiken verknüpft ist (vgl. Terlinden 1990; Notz 2004; Ostner 1982). Zahlreiche Theorien und Ansätze befassen sich dabei auf unterschiedlichen Ebenen (u. a. Makro-, Meso-, Mikroebene; Struktur- oder Handlungsebene; historische Perspektiven) mit den Bedingungsund Bedeutungszusammenhängen bestehender Geschlechterverhältnisse (vgl. Becker/ Kortendiek 2004; Becker-Schmidt/Knapp 2000), in denen für Männer vornehmlich entlohnte Erwerbsarbeit vorgesehen ist, während Frauen doppelt vergesellschaftet sind und damit einerseits der Erwerbsarbeit, aber vor allem der nicht entlohnten Haus- und Familienarbeit zugeordnet werden (vgl. Becker-Schmidt/Knapp 2000; Becker-Schmidt 2004). Diese Zuordnungen sind weiterhin mit einer geschlechtlich kodierten Hierarchisierung beider gesellschaftlicher Sphären und ihrer Akteur*innen verbunden, wobei die männlich konnotierte Erwerbsarbeit gegenüber der weiblich konnotierten Haus- und Familienarbeit als höherwertig gilt (vgl. Becker-Schmidt/Knapp 2000; Becker-Schmidt 2004). Als ein Begründungszusammenhang wird in diesem Kontext eine „männerbündische [...] Strukturierung der sozialen Welt“ (Becker-Schmidt/Knapp 2000: 61) angeführt. So konnte historisch betrachtet ,[d]as männliche Geschlecht [...] seine Maßstäbe bei der Abwertung der Hausarbeit als nicht marktvermittelter, unbezahlter und nicht professionalisierter Tätigkeit gegenüber bezahlter, öffentlicher und berufsförmiger geltend machen“" (Becker-Schmidt/Knapp 2000: 60).

Eine Trennung zwischen beruflichen und privaten Sphären des gesellschaftlichen Lebens findet sich auch in den benannten Zielen und Motivationen der befragten Nachwuchskräfte wieder. So spannen sechs der zehn befragten Nachwuchskräfte - Männer und Frauen gleichermaßen - im Interview ein Verhältnis zwischen beruflichen und privaten Zielen auf und nehmen damit eine Unterscheidung zwischen Erwerbstätigkeit und Privatleben vor. ${ }^{12}$

Dabei wird den privaten Zielen zum Teil explizit Priorität gegenüber dem Beruf eingeräumt, ohne dies weiter auszuführen. So benennt eine Person, dass ihr das Privatleben wichtiger sei als das berufliche Vorankommen, weshalb sie auch wenig Ehrgeiz und Ziele bezüglich einer bestimmten Position oder eines bestimmten Status habe. Eine weitere Person beschreibt, dass sie gerne dichter mit dem/der Ehepartner*in zusammen-

12 Vier der befragten Nachwuchskräfte gehen dagegen überwiegend oder ausschließlich auf Ziele und Motivationen ein, die sich auf die berufliche Tätigkeit beziehen; private Ziele werden hier nicht hervorgehoben. 
leben möchte und dass es ihr nicht wichtig sei, sich im Beruf mit anderen zu messen und sich neuen Herausforderungen zu stellen. Anerkennung im Beruf bzw. Karriere scheint hier eine eher untergeordnete Rolle zu spielen.

Ein weiterer Teil der befragten Nachwuchskräfte beschreibt ein Verhältnis zwischen beruflichen und privaten Zielen, das eher durch Kompromisse und Balance gekennzeichnet ist. Private Ziele werden genannt und teilweise bereits realisiert. Andere werden als langfristige Anliegen formuliert, auf die hingearbeitet werden soll; Zwischenlösungen werden praktiziert. Konkret wird etwa benannt, dass langfristig der Wunsch bestehe, mit dem/der Partner*in am selben Ort zu leben, nicht nur für die Arbeit zu leben oder auf einen Job hinzuarbeiten, der mehr Sicherheit verspreche, als es im wissenschaftlichen Bereich der Fall sei. Letzteres wird als Basis dafür beschrieben, sich privat entwickeln zu können.

Diese durch Prioritätensetzung und Balance charakterisierten Verhältnisse privater und beruflicher Ziele bzw. Motivationen werden überwiegend nicht als verunsichernd oder konflikthaft beschrieben. Vielmehr werden Kompromisse gefunden, eine Vereinbarkeit zu ermöglichen, oder die beruflichen Ziele werden untergeordnet, ohne dass dies als problematisch betrachtet wird. Die befragten Personen mit dieser Ausrichtung orientieren sich anscheinend souverän in diesem Spannungsfeld und treffen bewusst Entscheidungen.

Sichtbar wird damit bei den Nachwuchskräften ein deutliches Bekenntnis zum Privaten, wobei sie das Private im Interview vor allem mit Freund*innen, Familie und Beziehung, also mit sozialem Leben und sozialen Verbindlichkeiten in Verbindung bringen. Deutlich wird dabei, dass private und berufliche Ziele von denjenigen Nachwuchskräften, die im Interview auf beide Zielperspektiven eingehen, als mindestens gleichwertig beschrieben werden oder dass privaten Zielen ein höherer Wert beigemessen wird. Eine Trennung von Erwerbs- und privater Arbeit geht hier anscheinend nicht mit einer Praxis der Abwertung weiblich konnotierter Tätigkeiten bzw. gesellschaftlicher Sphären einher. Vielmehr scheint zumindest bei einigen der Befragten eher das Gegenteil der Fall zu sein. In den Aussagen eines Großteils der Nachwuchskräfte spiegeln sich somit sowohl eine ablehnende Haltung gegenüber einem ausschließlich auf Erwerbstätigkeit ausgerichteten Lebensstil als auch das Bestreben und der Versuch, dem Privatleben als Teil von Lebensqualität mehr Raum zu geben.

Das Private ist damit nicht mehr der Schatten des Öffentlichen und Beruflichen, die gesellschaftlich nicht anerkannte (und ökonomisch in Wert gesetzte) Regenerationszone, die als weiblich kodiert alle dort stattfindenden Tätigkeiten und dafür notwendigen Kompetenzen entfachlicht und aus dem ökonomischen Kalkül ausklammert, sie zugleich jedoch als heimliche Ausgangsbasis unentgeltlich und dauerhaft verfügbar einbezieht und ausbeutet (vgl. dazu Hofmeister/Katz/Mölders 2013; Teubner 2004), im Gegenteil. Das Private in dieser Deutung ist machtvoll, wird aktiv gewählt, ist gemeinschaftsorientiert konzipiert und Ausdruck von Selbstfürsorge. Es steht damit für eine bewusste Lebensgestaltung in gegenseitiger Verantwortungs- und Aufgabenteilung.

Die Positionierung eines Großteils der Nachwuchskräfte für das Private ist darüber hinaus als emanzipatorisch zu verstehen, insofern damit ein Widerstand gegenüber dauernder Verfügbarkeit, gegenüber einer totalitären Nutzenmaximierung und Effizienzorientierung einhergeht - wie sie Wissenschaft und Bildung in neoliberalen Zeiten 
kennzeichnet (Lösch 2008). Ein Trend, den laut Hofbauer sowohl die Forschung zur Krise moderner Männlichkeit als auch die Forschung zu erwerbstätigen Frauen bereits aufgegriffen hat (Hofbauer 2004: 59). Erstere stellt diesbezüglich laut Hofbauer eine „,reflexive Distanzierung“ (Hofbauer 2004: 59) von Männern gegenüber einer einseitigen Fokussierung auf Erwerbsarbeit fest, die u. a. mit der Suche nach alternativen Anerkennungskontexten angesichts unsicherer Erwerbsarbeitsverhältnisse sowie mit dem Wunsch nach einer stärkeren Ausbalancierung beruflicher und privater Bedürfnisse in Verbindung gebracht wird (Hofbauer 2004: 59). Bezogen auf erwerbstätige Frauen weist Hofbauer auf Studien von Simpson (1998) und Völker (1999) hin, die widerständige Praktiken von Frauen gegenüber beruflichen Anforderungen skizzieren: Von männlichen Kollegen gelebte oder betrieblich geforderte und für den beruflichen Kontext Vorteil bringende dauerhafte Verfügbarkeit oder extreme Überstundenleistungen werden bewusst abgelehnt und verweigert (Hofbauer 2004: 58f.).

Die Spitzenkräfte konstruieren ein solches Verhältnis zwischen privaten und beruflichen Zielen im Interview nicht. Sie gehen fast ausschließlich auf berufliche Ziele ein, die wie bei den befragten Nachwuchskräften an das Leitbild einer nachhaltigen Entwicklung gekoppelt sind. Private Ziele werden im Interview nur gestreift, wenn etwa benannt wird, dass nachhaltige Entwicklung für einige der Befragten auch privates Ziel ist, Arbeit an nachhaltigkeitsrelevanten Inhalten in die private Zeit gelegt wird und persönliche Werte mit der beruflichen Tätigkeit vereinbar sein müssen, oder es wird eine hohe Arbeitsbelastung kritisiert. Das Privatleben wird hier also wenig angesprochen - unabhängig vom biologischen Geschlecht der Befragten. Zur Sprache kommen fast ausschließlich Aspekte, die den Bereich der Erwerbsarbeit betreffen. Inwieweit damit tatsächlich eine Priorisierung der Erwerbsarbeit gegenüber dem Privatleben oder eine Abwertung des Privatlebens und damit verbundenen Tätigkeiten einhergehen, bleibt an dieser Stelle jedoch offen.

Insgesamt betrachtet wird somit für den Kontext wissenschaftlicher Tätigkeitsfelder im Bereich Nachhaltigkeit sichtbar, dass sich ein Großteil der befragten Nachwuchskräfte im Interview sowohl mit der weiblich konnotierten Sphäre des Privaten als auch mit der männlich konnotierten Erwerbsarbeit identifiziert, das Private dabei explizit einfordert und ihm einen hohen Wert beimisst. Die Spitzenkräfte fokussieren demgegenüber im Interview die Erwerbsarbeit und damit männlich konnotierte Aspekte. Es deutet sich also eine positionsbedingte Trennungslinie zwischen Spitzen- und Nachwuchspositionen an.

\subsection{Bedeutungen und Bewertungen von Aspekten fachlicher und sozial- kommunikativer Kompetenz von Spitzen- und Nachwuchskräften: Grenzverschiebungen bei geschlechterkodierten Hierarchisierungen}

In den Interviews betonen alle Spitzenkräfte, dass vertiefte Fachexpertise und das Einbringen von dafür erforderlichen Fähigkeiten in ihrem Arbeitsalltag eine untergeordnete Rolle einnehmen. Vielmehr bringen sie Überblickswissen, Strukturierungs- und Prozessgestaltungsfähigkeiten sowie sozial-kommunikative Kompetenzen (z. B. zur Motivation und Einbindung der Mitarbeiter*innen) ein und schaffen damit (atmosphärische) Arbeitsverhältnisse, die der Optimierung der Leistungs- und Funktionsfähigkeit des Teams dienen. 
Die Notwendigkeit der Teamarbeit wird nahezu ausschließlich instrumentell begründet, z. B., um zu einer nachhaltigen Entwicklung beizutragen oder Aufgaben erfüllen und Produkte erzielen zu können. Die Spitzenkräfte beschreiben sich in ihrer Position als funktional-inhaltlich angewiesen auf verschiedene disziplinäre Sichtweisen und Kenntnisse sowie deren Zusammendenken. Beziehungsorientierung als ein wesentlicher Bestandteil sozial-kommunikativer Fähigkeiten dient ihnen dazu, kooperative Arbeitsstrukturen und -kulturen herzustellen, die den Zugang zu fachlicher Expertise und deren Vermittlung in die Organisation sowie nach außen ermöglichen. Solche Bedingungen werden von den interviewten Spitzenkräften als essenziell bedeutsam für ihre Funktion und ihren fachlichen Status beschrieben, Beziehungsarbeit und Sozial-Kommunikatives werden damit stark instrumentell verstanden ${ }^{13}$ und entsprechend ergebnisorientiert sowie zur Realisierung von Fachlichkeit und Professionalität eingesetzt.

Die befragten Nachwuchskräfte entwerfen ein Teamarbeitsverständnis, das von klar hierarchisch über dialogisch und kooperationsorientiert bis hin zur Vorstellung eines gleichberechtigten Miteinanders reicht. Unabhängig davon konzipieren bis auf wenige Ausnahmen alle Nachwuchskräfte soziales Miteinander und Beziehungsorientierung in beruflichen Kontexten als existenzielles Bedürfnis. Sie verfolgen damit kein unmittelbares Anliegen, sondern beschreiben ihre Arbeitszusammenhänge als Prozesse, in denen das fachlich-methodische Know-how mit dem sozial-kommunikativen Kompetenzbereich verwoben ist und Gemeinschaft unmittelbar Sinn und Orientierung stiftet. Sie betten die für ihre Arbeitspraxis formulierte Beziehungsorientierung wie selbstverständlich in das Fachliche ein, denken sie mit ihm zusammen. Damit verschieben sich die Grenzen und Trennungen zwischen dem, was unter sozial-kommunikativen und fachbezogenen Kompetenzen gefasst wird. Denn soziale Aspekte werden nicht nur als in der Kommunikation und Interaktion im Prozess und den Arbeitsbeziehungen bedeutsam konstruiert. Sie werden auch auf die Untersuchungsobjekte selbst bezogen (z. B. spezifische Teile der Natur als Beziehungsobjekt), gelten für das Private und das Berufliche gleichermaßen. Folgende vier Begründungen für eine Beziehungsorientierung können aus den Aussagen der Nachwuchskräfte herausgefiltert werden:

- $\quad$ ein essenzielles Bedürfnis nach Erleben von Gemeinschaft, um Orientierung und Sicherheit zu gewinnen (u. a. über die anderen als Korrektiv und Reflexionsfolie für das eigene Verhalten und die eigenen Leistungen),

- die Rücksichtnahme auf und die Berücksichtigung von anderen Interessen (Herstellen eines Interessensausgleichs) und Befindlichkeiten (ethische Gründe),

- $\quad$ anderen darüber Arbeitsaufträge besser verständlich zu machen bzw. durch Kooperation und den Austausch von Daten und Fakten gegenseitigen Nutzen zu ziehen und/ oder konfliktreiche, schwierige Situationen zu bewältigen (strategische Gründe), ${ }^{14}$

- $\quad$ ein altruistisches Engagement, das sich nicht am Verhalten anderer ausrichtet, sondern ggf. damit verbundene Nachteile bewusst in Kauf nimmt.

13 Lediglich eine Spitzenkraft beschreibt das diskursive Zusammenarbeiten an sich als bereichernd und glücksbringend.

14 Von einer Person wird ein bewusstes soziales Unterordnungsverhalten, Stillhalten, Sich-Zurücknehmen als Anpassungsstrategie beschrieben. 
Diese Ergebnisse zum Verhältnis von Beziehungsorientierung als einem Merkmal sozial-kommunikativer Kompetenz und Fachlichkeit weisen Bezüge zu dem in Kapitel 3.1 eingeführten, von Seiten feministischer Forschung kritisierten Trennungsparadigma auf. Nach dessen Logik werden soziale Stärken und vermittelnde, kommunikative Fähigkeiten, Emotionalität, Subjektivität und Intuition noch immer als etwas Frauen oder der weiblichen Sphäre Zugehöriges gedacht. Fachlichkeit, Sachlichkeit sowie Technikverständnis hingegen werden stärker mit Männern/männlichen Bereichen assoziiert (Wajzman 1996; Faulkner 2000; Lucht/Paulitz 2008). Diese geschlechterkonnotierte unterschiedliche Bewertung von Fähigkeiten, wie sie insbesondere bei naturwissenschaftlich-technisch geprägten und damit in den meisten umweltbezogenen Berufsfeldern nachgewiesen werden kann (z. B. Scheich 1993; Wiesner 2002), findet sich auch in den Aussagen von zwei Befragten. So gibt eine Spitzenkraft an, in Konflikten gezielt Fähigkeiten aus dem fachlich-methodischen Bereich einzusetzen, um so durch Fachlichkeit eine sachliche Ebene einzubringen. Dies sei insbesondere für weibliche Führungskräfte wichtig, da diese eher der Gefahr ausgesetzt seien, nicht so ernst genommen, stattdessen „als irgendein emotionsgesteuertes Wesen“ wahrgenommen zu werden. Der Rekurs auf rationale Fach- und Sachlichkeit fungiert hier weiterhin als Chiffrierung für Autorität und dient der Absicherung von Status.

In den Aussagen von Spitzen- und Nachwuchskräften zur Bedeutung von Fachlichkeit ist erkennbar, dass das soziale Miteinander, die Beziehungsarbeit - die eher dem Privaten zugeordnet wird und komplementär zum Öffentlichen steht - im Kontext der Erwerbsarbeit einen hohen Stellenwert erhält. Im Ergebnis zeigen sich jedoch positionsbezogene Unterschiede: Spitzenkräfte beziehen ihre sozial-kommunikativen Fähigkeiten explizit auf die Teamprozesse, die sie deutlich in den Dienst der Aufgabe stellen. Essenzielle oder altruistische Erwägungen spielen weniger eine Rolle als berufsbezogene strategische Nützlichkeitserwägungen sowie Loyalität der Arbeitgeberin/dem Arbeitgeber bzw. der Aufgabe gegenüber. Das heißt: Der Einsatz dieser Fähigkeiten festigt Macht- und Führungsposition. Nachwuchskräfte zielen mit ihrem Kompetenzeinsatz aus diesem Bereich dagegen einerseits zwar auch auf die Realisierung einer guten fachlichen Zusammenarbeit. Es geht ihnen andererseits aber insbesondere um ein existenzielles „In-Beziehung-Sein“, um eine neue Qualität des Arbeitsprozesses und der Arbeit an sich sowie ihrer Wirkung im Team.

\section{Perspektiven einer Neuorientierung in wissenschaftlichen Tätigkeitsfeldern im Bereich Nachhaltigkeit}

Vor dem Hintergrund der vorangegangenen Ausführungen zur Bedeutung von Privatleben und Erwerbstätigkeit sowie zum Verhältnis von Fachlichkeit und Beziehungsorientierung in Arbeitskontexten bei Nachwuchs- und Spitzenkräften ergeben sich Hinweise auf weiteren Forschungsbedarf.

Indem der Großteil der befragten Nachwuchskräfte das soziale Miteinander im Zentrum und als existenzielles Element von Arbeitskontexten konstruiert, platzieren sie einen Aspekt in der Erwerbstätigkeit, der klassischerweise eher im Privaten veror- 
tet wird. Die befragten Nachwuchskräfte scheinen somit nicht nur dem Privatleben als komplementärem Element zur Erwerbstätigkeit einen hohen Wert beizumessen, sondern es besteht auch der Wunsch danach, Beziehungsorientierung als essenziellen Teil von Lebensqualität in nachhaltigkeitsorientierten Arbeitskontexten zu realisieren.

So stellt sich zunächst die Frage, was der identifizierte Stellenwert des Privaten für Menschen und Karrieren in wissenschaftlichen Tätigkeitsfeldern im Bereich Nachhaltigkeit bedeutet. Betrachtet man das Bedürfnis zumindest eines Teils der Nachwuchskräfte nach Raum für Privatheit neben dem Erwerbsleben und für Aspekte des Sozialen und In-Beziehung-Seins im Erwerbsleben einerseits und die deutliche Fokussierung der Spitzenkräfte auf Erwerbsarbeit und auf eine instrumentelle Nutzung von Gemeinschaft andererseits, deutet sich eine Barriere hinsichtlich des Erreichens von Spitzenpositionen an. Es könnte die fehlende Möglichkeit in Spitzenpositionen sein, ein individuell ausbalanciertes Verhältnis zwischen Erwerbsarbeit und Privatleben zu gestalten, die Menschen, die sich sowohl mit der männlich konnotierten Sphäre der Erwerbsarbeit als auch mit der weiblich konnotierten des Privaten identifizieren, daran hindert, in Spitzenpositionen zu gelangen.

Hinweise darauf, dass Erwerbsarbeit ein solches individuell ausbalanciertes Verhältnis nicht selbstverständlich ermöglicht, liefert beispielsweise Funken im Rekurs auf Ansätze von Acker zu Organisation und Geschlecht, wenn sie beschreibt, dass ,,[d]ie Regeln von Organisationen [...] getrennte Sphären [erzeugen] - hier Erwerbsarbeit und dort Privatleben -, wobei explizit und implizit verlangt wird, dass die Erwerbsarbeit absolute Priorität einnimmt“ (Funken 2004: 23). Sie führt weiter aus, dass ,dies [...] nach Acker zur Unterscheidung von zwei Sorten Beschäftigten [führt]: denjenigen, die diese Regeln befolgen (können) und diejenigen, die aufgrund von Familien- und Reproduktionsarbeiten diese Regeln nicht oder nur eingeschränkt befolgen (können)“ (Funken 2004: 23). Es ,[entpuppt sich] vor dem Hintergrund fortbestehender Arbeitsteilung zwischen den Geschlechtern [...] der (!) abstrakte Erwerbsarbeitende bei der Dekonstruktion der organisationalen Sub-Struktur in der Regel immer noch als Mann“ (Funken 2004: 23).

Diesbezüglich gilt es nun zu klären, inwieweit insbesondere die Priorisierung der Erwerbsarbeit im betrachteten Kontext möglicherweise für Spitzenpositionen vorausgesetzt wird, während sich in Nachwuchspositionen diesbezüglich ein Wandel abzeichnet. Dominieren im Bereich von Nachwuchspositionen in wissenschaftlichen Tätigkeitsfeldern im Nachhaltigkeitsbereich möglicherweise Strukturen (hier im Sinne von Giddens' Strukturationstheorie (1995) verstanden als Struktur und Handeln), die eine Auflösung von Prozessen der Auf- und Abwertung, die mit einer geschlechtsspezifischen Trennung zwischen Erwerbsarbeit und Privatleben verbunden sind, bzw. eine Ausstattung des weiblich konnotierten Privaten mit Macht eher ermöglichen oder befördern, als es bei Spitzenpositionen der Fall zu sein scheint?

Ein weiteres Hindernis könnte auch die starke Nutzen- und Verwertungsausrichtung sein, die hinter der Indienstnahme von Sozialität bei Spitzenkräften steht. Nachwuchskräfte konzipieren demgegenüber soziales Miteinander und Beziehungsorientierung als existenziellen und konstitutiven Bestandteil fachlicher Arbeitskontexte. Das Einnehmen einer Spitzenposition wird dann mit einem existenziellen Verlust verbunden. Dies erscheint ein umso schwerwiegenderes Hindernis für Positionierungen in nachhaltigkeitsbezogenen Tätigkeitsfeldern, als der Beziehungsorientierung und einem In-Beziehung- 
Sein in einem jüngst im Rahmen des NaGe-Projektes diskursiv erstellten genderorientierten Anforderungsprofil für Nachhaltigkeitskompetenzen (z. B. Thiem/Katz 2014) ein sehr hoher Stellenwert als auszubildende Teilkompetenzen attestiert wurde.

Und zu guter Letzt stehen einige geschlechterrelevante strukturelle Bedingungen dem Aufstieg von Menschen mit einer starken Beziehungsorientierung und Betonung des Privaten entgegen. Bisher sind es noch vorwiegend Männer, die in Organisationen Stellenprofile und -aufgaben definieren und damit Stellen auch in ihrem Sinne besetzen können (vgl. Wilz 2002: 67ff.). Das heißt: Die weiterhin existente geschlechterkodierte Abwertung des sozial-kommunikativen gegenüber dem fachlich-methodischen Bereich wirkt auf Personalentscheidungen ein und befördert tendenziell die Reproduktion geschlechterhierarchischer Segregation. Dies wird zusätzlich unterstützt durch die noch immer stark sektoral gegliederten Organisationseinheiten in den meisten nachhaltigkeitsorientierten Institutionen, die eine teamübergreifende Zusammenarbeit sowie den Aufbau kooperativer und integrativer Strukturen erschweren.

Die starke Stellung des Privaten bei einem Großteil der befragten Nachwuchskräfte kann umgekehrt jedoch auch Potenzial für Veränderungen im Bereich von Nachwuchsund Spitzenpositionen in sich tragen, wenn sich die Forderung nach Privatleben neben und im Erwerbsleben bei Nachwuchskräften verstärkt und verstetigt und möglicherweise auch auf den Bereich von Spitzenpositionen übergreift. Auch dies gilt es weiter zu untersuchen.

\section{Literaturverzeichnis}

Becker, Egon \& Jahn, Thomas. (Hrsg.). (2006). Soziale Ökologie. Grundzüge einer Wissenschaft von den gesellschaftlichen Naturverhältnissen. Frankfurt/Main, New York: Campus.

Becker, Ruth \& Kortendiek, Beate.(Hrsg.). (2004). Handbuch Frauen-und Geschlechterforschung. Theorie, Methoden, Empirie. Wiesbaden: VS Verlag.

Becker-Schmidt, Regina. (2004). Doppelte Vergesellschaftung von Frauen: Divergenzen und Brückenschläge zwischen Privat- und Erwerbsleben. In Ruth Becker \& Beate Kortendiek (Hrsg.), Handbuch Frauen- und Geschlechterforschung. Theorie, Methoden, Empirie (S. 62-71). Wiesbaden: VS Verlag.

Becker-Schmidt, Regina. (2007 [1993]). Geschlechterdifferenz - Geschlechterverhältnis: soziale Dimensionen des Begriffs , Geschlecht' ${ }^{\star}$. In Sabine Hark (Hrsg.), Dis/Kontinuitäten. Feministische Theorien (2. Auflage) (S. 115-127). Wiesbaden: VS Verlag.

Becker-Schmidt, Regina \& Knapp, Gudrun-Axeli. (2000). Feministische Theorien. Zur Einführung. Hamburg: Junius.

Blättel-Mink, Brigit; Briken, Kendra \& Rau, Alexandra. (2010). Grenzen des Gender Mainstreaming? Professionalisierung der akademischen (Selbst)Verwaltung und die Beharrlichkeit von ,, doing gender “. Projektbericht im Rahmen der Förderung kleiner Frauen- und Genderforschungsprojekte. Frankfurt/Main. Zugriff am 4. Juli 2014 unter www. fb03.unifrankfurt.de/45724080/Rau_2010_Grenzen_Gender_Mainstreaming.pdf.

Bundesministerium für Umwelt, Naturschutz und Reaktorsicherheit. (Hrsg.). (o. J.). Umweltpolitik. Konferenz der Vereinten Nationen für Umwelt und Entwicklung im Juni 1992 in Rio de 
Janeiro - Dokumente. Agenda 21. Zugriff am 15. April 2014 unter http://www.bmu.de/ fileadmin/bmu-import/files/pdfs/allgemein/application/pdf/agenda21.pdf.

Erpenbeck, John. (2004). KODE ${ }^{\circledR}$ im Tableau quantitativer, qualitativer und komparativer Kompetenzmessverfahren in Deutschland. In Volker Heyse, John Erpenbeck \& Horst Max (Hrsg.), Kompetenzen erkennen, bilanzieren und entwickeln (S. 118-131). Münster u. a.: Waxmann.

Erpenbeck, John \& Rosenstiel, Lutz von. (Hrsg.). (2007 [2003]). Handbuch Kompetenzmessung. Erkennen, verstehen und bewerten von Kompetenzen in der betrieblichen, pädagogischen und psychologischen Praxis. Stuttgart: Schaeffer-Poeschel.

Faulkner, Wendy. (2000). The Power and the Pleasure: How does Gender 'stick' to Engineers? Science, Technology \& Human Values, 25(1), 87-119.

Funken, Christiane. (2004). Zu Chancen und Risiken von (in)formellen Organisationsstrukturen für die Karrieren von Frauen und Männern. In Ursula Pasero \& Birger P. Priddat (Hrsg.), Organisationen und Netzwerke: Der Fall Gender (S. 13-44). Wiesbaden: VS Verlag.

Giddens, Anthony. (1995). Die Konstitution der Gesellschaft. Frankfurt/Main, New York: Campus.

Glaser, Barney G. \& Strauss, Anselm L. (1998). Grounded Theory. Strategien qualitativer Forschung. Bern u. a.: Huber.

Heyse, Volker. (2010). Verfahren zur Kompetenzermittlung und Kompetenzentwicklung. In Volker Heyse, John Erpenbeck \& Stefan Ortmann (Hrsg.), Grundstrukturen menschlicher Kompetenz. Praxiserprobte Konzepte und Instrumente (S. 55-147). Münster u. a.: Waxmann.

Heyse, Volker \& Erpenbeck, John. (2010). Qualitätsanforderungen an $\mathrm{KODE}^{\circledR}$. In Volker Heyse, John Erpenbeck \& Stefan Ortmann (Hrsg.), Grundstrukturen menschlicher Kompetenzen. Praxiserprobte Konzepte und Instrumente (S. 21-54). Münster u. a.: Waxmann.

Heyse, Volker; Erpenbeck, John \& Ortmann, Stefan. (Hrsg.). (2010). Grundstrukturen menschlicher Kompetenz. Praxiserprobte Konzepte und Instrumente. Münster u. a.: Waxmann.

Hofbauer, Johanna. (2004). Distinktion - Bewegung an betrieblichen Geschlechtergrenzen. In Ursula Pasero \& Birger P. Priddat (Hrsg.), Organisationen und Netzwerke: Der Fall Gender (S. 45-64). Wiesbaden: VS Verlag.

Hofmeister, Sabine; Katz, Christine \& Mölders, Tanja. (2013). Geschlechterverhältnisse und Nachhaltigkeit. Die Kategorie Geschlecht in den Nachhaltigkeitswissenschaften. Opladen: Barbara Budrich.

Katz, Christine \& Thiem, Anja. (2011). Does Gender matter in gaining advantage in scientific fields of sustainability in Germany? A bibliographical approach. In Angela Calvo, Graziella Fornengo, Rachel Raus \& Flavia Zucco (Hrsg.), World Wide Women: Globalizzazione, Generi, Linguaggi, Volume 1, Selected Papers (S. 135-143). CIRSDe, Università degli Studi di Torino. Zugriff am 15. April 2014 unter http://aperto.unito.it/bitstream/2318/790/1/primo_ volume_WWW.pdf.

Katz, Christine \& Thiem, Anja. (2013). Führen Nachhaltigkeitskompetenzen an die Spitze? Geschlechterrelevante Faktoren des Gelingens und Scheiterns. In Felizitas Sagebiel, Ulla Hendrix, Christina Schrettenbrunner \& Uta von Winterfeld (Hrsg.), Organisationskultur und Macht - Veränderungspotenziale von Führungsfrauen in Umwelt und Technik (S. 203-220). Berlin u. a.: LIT Verlag.

Katz, Christine; Thiem, Anja; Moths, Katharina \& Heilmann, Sebastian. (2014). Nachhaltigkeitskompetenzim Spannungsfeld zwischen Kontextabhängigkeit und Perspektiverweiterung. In Ulrike Busolt, Sabrina Weber, Constantin Wiegel \& Wiebke Kronsbein (Hrsg.), 
Karriereverläufe in Forschung und Entwicklung - Bedingungen und Perspektiven im Spannungsfeld von Organisation und Individuum (S. 307-330). Berlin: Logos.

Kreckel, Reinhard. (2005). Mehr Frauen in akademischen Spitzenpositionen: Nur noch eine Frage der Zeit? Zur Entwicklung von Gleichheit und Ungleichheit zwischen den Geschlechtern. Zugriff am 4. Juli 2014 unter www2.soziologie.uni-halle.de/emeriti/kreckel/docs/genus2005korr2.pdf.

Kreuser,Karl.(2010). Tolerieren, Unterscheiden, Verändern! Strategisches Kompetenzmanagement mit KODE unter Diversity-Aspekten am Beispiel von Gender. Heidelberg: Carl Auer.

Lösch, Bettina. (2008). Politische Bildung in Zeiten neoliberaler Politik: Anpassung oder Denken in Alternativen. In Christoph Butterwegge, Bettina Lösch \& Ralf Ptak (Hrsg.), Neoliberalismus. Analysen und Alternativen (S. 335-354). Wiesbaden: VS Verlag.

Lucht, Petra \& Paulitz, Tanja. (Hrsg.). (2008). Recodierungen des Wissens. Stand und Perspektiven der Geschlechterforschung in Naturwissenschaft und Technik. Frankfurt/Main: Campus.

Meuser, Michael \& Nagel, Ulrike. (1991). ExpertInneninterviews - vielfach erprobt, wenig bedacht. Ein Beitrag zur qualitativen Methodendiskussion. In Detlef Garz \& Klaus Kraimer (Hrsg.), Qualitativ empirische Sozialforschung (S. 441-471). Opladen: Westdeutscher Verlag.

Meuser, Michael \& Nagel, Ulrike. (2004). ExpertInneninterview. In Ruth Becker \& Beate Kortendiek (Hrsg.), Handbuch Frauen- und Geschlechterforschung. Theorie, Methoden, Empirie (S. 326-329). Wiesbaden: VS Verlag.

Morrison, Ann M.; White, Randall P. \& Van Velsor, Ellen. (1987). Breaking the glass ceiling: Can women reach the top of America's largest corporations? Boston: Addison-Wesley Pub Co.

Müller, Ursula. (2013). Zwischen Licht und Grauzone: Frauen in Führungspositionen. In Ursula Müller, Birgit Riegraf \& Sylvia Marlene Wilz (Hrsg.), Geschlecht und Organisation (S. 469494). Wiesbaden: VS Verlag.

Notz, Gisela. (2004). Arbeit: Hausarbeit, Ehrenamt, Erwerbsarbeit. In Ruth Becker \& Beate Kortendiek (Hrsg.), Handbuch Frauen- und Geschlechterforschung. Theorie, Methoden, Empirie (S. 420-428). Wiesbaden: VS Verlag.

Osterloh, Margit \& Littmann-Wernli, Sabina. (2000). Die „gläserne Decke“: Realität und Widersprüche. In Sibylle Peters \& Norbert Bensel (Hrsg.), Frauen und Männer im Management. Diversity in Diskurs und Praxis (S. 123-139). Wiesbaden: Gabler.

Ostner, Ilona. (1982). Beruf und Hausarbeit. Frankfurt/Main: Campus.

Scheich, Elvira. (1993). Naturbeherrschung und Weiblichkeit. Denkformen und Phantasmen der modernen Naturwissenschaften. Pfaffenweiler: Centaurus.

Simpson, Ruth. (1998). Presenteeism, power and organizational change: Long hours as a career barrier and the impact on the working lives of women managers (special issue). British Journal of Management, 9(3), 37-50.

Terlinden, Ulla. (1990). Gebrauchswirtschaft und Raumstruktur: ein feministischer Ansatz in der soziologischen Stadtforschung. Stuttgart: Silberburg-Verlag.

Teubner, Ulrike. (2004). Beruf: Vom Frauenberuf zur Geschlechterkonstruktion im Berufssystem. In Ruth Becker \& Beate Kortendiek (Hrsg.), Handbuch Frauen- und Geschlechterforschung. Theorie, Methoden, Empirie (S. 429-436). Wiesbaden: VS Verlag.

Thiem, Anja \& Katz, Christine. (2014). Nachhaltigkeitskompetenz und Gender - Anforderungen und Hindernisse für Spitzen- und Nachwuchskräfte in nachhaltigkeitsorientierten Tätigkeitsbereichen. In Christine Katz, Sebastian Heilmann, Anja Thiem, Lea Koch, Katharina Moths, 
Sabine Hofmeister (Hrsg.), Nachhaltigkeit anders denken. Veränderungspotenziale durch Geschlechterperspektiven. Wiesbaden: VS Verlag [im Erscheinen].

Völker, Susanne. (1999). Erwerbsorientierungen und betriebliche Transformation. Selbstverortungen und Handlungsstrategien ostdeutscher Frauen bei der Deutschen Bahn AG. In Hildegard Maria Nickel, Susanne Völker \& Hasko Hüning (Hrsg.), Transformation - Unternehmensreorganisation - Geschlechterforschung (S. 205-226). Opladen: Leske + Budrich. Wajzman, Judy. (1996). Feminism confronts technology. Cambridge: Polity.

Wiesner, Heike. (2002). Die Inszenierung der Geschlechter in den Naturwissenschaften. Wissenschafts- und Genderforschung im Dialog. New York u. a.: Campus.

Wilz, Sylvia Marlene. (2002). Organisation und Geschlecht - strukturelle Bindungen und kontingente Kopplungen. Opladen: Leske + Budrich.

Winterfeld, Uta von. (2013). Macht für Nachhaltigkeit. In Felizitas Sagebiel (Hrsg.), Organisationskultur und Macht (S. 177-201). Berlin: LIT Verlag.

\section{Zu den Personen}

Anja Thiem, Dr. phil., wissenschaftliche Mitarbeiterin in verschiedenen Forschungsprojekten im Lehr- und Forschungsgebiet Umweltplanung, KODE®- und KODE®X-Lizenztrainerin für Kompetenzdiagnostik und -entwicklung, Koordination des Projektes „Nachhaltigkeit und Geschlecht $(\mathrm{NaGe})$ “. Arbeitsschwerpunkte: strategisches Qualitätsmanagement, nachhaltige Raumentwicklung, ländliche Räume, Geschlechterverhältnisse und Nachhaltigkeit, Nachhaltigkeitskompetenzen sowie Nachwuchsförderung und Mentoring.

Kontakt: Leuphana Universität Lüneburg, Scharnhorststraße 1, 21335 Lüneburg

E-Mail: thiem@uni.leuphana.de

Christine Katz, Dr. rer. nat., Tätigkeit in der Ökosystemforschung und der wissenschaftlichen Politikberatung, Forschungsprojektleitung zum Natur-/Tätigkeitsverständnis bei Umweltakteur*innen und zu Nachhaltigkeit und Gender. Arbeitsschwerpunkte: Genderexpertisen, Gendertraining im Bereich der Umwelt-/Nachhaltigkeitspolitik, Klimafolgenforschung, Gender- und Nachhaltigkeitskompetenzen.

Kontakt: Leuphana Universität Lüneburg, Scharnhorststraße 1, 21335 Lüneburg

E-Mail: waldfrauen@uni.leuphana.de

Katharina Moths, Dipl.-Umweltwissenschaftlerin, wissenschaftliche Mitarbeiterin am Institut für Nachhaltigkeitssteuerung an der Leuphana Universität Lüneburg, Bereich Umweltplanung im Rahmen des Projekts „Nachhaltigkeitskompetenz und Geschlecht“. Arbeitsschwerpunkte: Zusammenhang von Organisation, Nachhaltigkeit und Geschlecht.

Kontakt: Leuphana Universität Lüneburg, Scharnhorststraße 1, 21335 Lüneburg

E-Mail: moths@uni.leuphana.de 